\title{
Renovascular Hypertension in Chronic Hemodialytic Patient
}

\author{
Sri Lestari*, Heru Prasanto, Iri Kuswadi \\ Department of Internal Medicine, Division of Nephrology, Faculty of Medicine, Public Health and Nursing, Universitas Gadjah \\ Mada, Dr. Sardjito Hospital, Yogyakarta, Indonesia
}

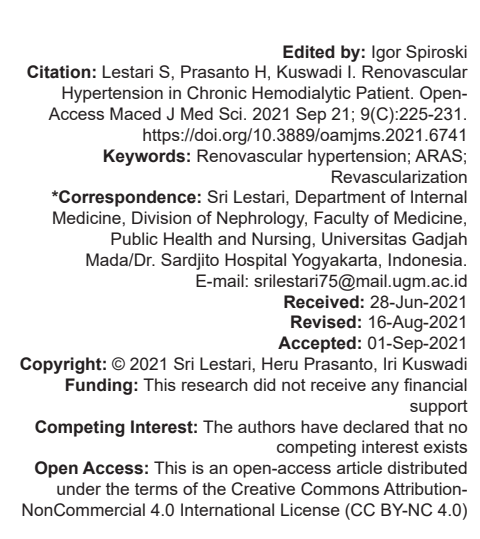

\section{Introduction}

Chronic kidney disease (CKD) and hypertension are chronic conditions that are closely related. As either a cause or a consequence of CKD, hypertension is very common in patients with CKD. The kidneys are known to have a fundamental role in the regulation of blood pressure. Animal studies have consistently shown that injury to the kidneys causes hypertension. Human studies have shown an association between a decrease in the number of nephrons and a decrease in the glomerular filtration rate (GFR) with an increased risk of hypertension. Hypertension is a major contributor to the development of CKD [1].

The prevalence of hypertension in Indonesia is quite high (34.11\%). The National Basic Health Research (Riskesdas) Report 2018 of Indonesia, revealed that the prevalence of hypertension by age group was $45.3 \%$ at the age of $45-54$ years, $55.2 \%$ at the age of 55-64 years [2]. According to 2018 Indonesian Renal Registry data, the most cause of patients with CKD Stage 5 on dialysis is hypertension (36\%) and diabetic nephropathy or diabetic kidney disease $(28 \%)$ [3]. Estimates of the prevalence, treatment, and control of hypertension in chronic dialysis patients vary widely. This variability arises, in large part because of the differences in the definitions used to diagnose hypertension and the methods of measuring blood pressure in the various studies [4]. It was reported that in Indonesia, hypertension is the most comorbid disease in CKD patients on dialysis (51\%) [3].

Hypertension in CKD on dialysis patients is common and often uncontrolled. Additional tests are needed to determine other causes of hypertension if secondary hypertension is suspected. The priority of examination should be directed to the suspected cause, based on signs or symptoms [5].

Renovascular hypertension is the most common cause of secondary hypertension and the prototype of angiotensin-dependent hypertension, and caused by renal artery occlusion. Occlusion can be caused by stenosis. Renal artery stenosis (RAS) is a substantial clinical problem, especially in resource-limited countries. In general, RAS is categorized into atherosclerotic RAS (ARAS) (90\%) and non-ARAS (10\%) [6].

Diagnosis and treatment of RAS is very important. It can accelerate the achievement of blood pressure targets, reduce the risk and complications due to hypertension, and in some cases, secondary hypertension can be cured. The history of the treatment of renovascular hypertension also varies, starting in the 1960s when the first surgery for 
revascularization was performed. The most widely performed treatment is percutaneous transluminal renal angioplasty (PTRA).

\section{Case Report}

A 52-year-old woman with CKD has been undergoing hemodialysis for 2 years. The etiology of CKD is diabetes mellitus. She has diabetes in the past 11 years and hypertension in the past 5 years. Hypertension that was previously well controlled for 2 years required an increase in antihypertensive therapy from 2 to 4 drugs in recent months. The patient underwent routine outpatient hemodialysis with left arm AV fistula access and a Braun Dialog $+B$ machine. Dialysis sessions were $5 \mathrm{~h}$ twice weekly with polysulfone hollow fiber dialyzers (B Braun) and using bicarbonate-based dialysate. The dialysate calcium level is $1.9 \mathrm{mmol} / \mathrm{L}$, the dialysate magnesium level is $1 \mathrm{mmol} / \mathrm{L}$. The blood flow rate varies between 200 and $250 \mathrm{~mL} / \mathrm{min}$. The dialysate flow rate was constant $500 \mathrm{~mL} / \mathrm{min}$. Dialysis adequacy was measured by $\mathrm{Kt} / \mathrm{v}$, that is, 1.24-1.41. The average interdialytic weight gain was $2 \mathrm{~kg}$.

The physical examination revealed that patient was good and composmentis. While, the results of anthropometric examination were weight of $50 \mathrm{~kg}$, height of $152 \mathrm{~cm}$, and BMI of $21.64 \mathrm{~kg} / \mathrm{m}^{2}$. The blood pressure was 180/90 $\mathrm{mmHg}$, pulse $79 \mathrm{bpm}$, respiration 20/min, and temperature $36.7^{\circ} \mathrm{C}$. Conjunctiva looks anemic, cardiomegaly, lungs within normal limits, no ascites, and edema in the extremities. The laboratory results were hemoglobin $9.5 \mathrm{~g} / \mathrm{dL}(13-18 \mathrm{~g} / \mathrm{dL})$ and creatinine $6.29 \mathrm{mg} / \mathrm{dL}(0.7-1.2 \mathrm{mg} / \mathrm{dL})$. On renal artery duplex ultrasound examination, the acceleration time of the right main renal artery was 147.65 ms, Peak Systolic Velocity (PSV) $31.9 \mathrm{~cm} / \mathrm{s}$. RI 0.69 and left main renal artery acceleration time $120.81 \mathrm{~ms}$, PSV $16.9 \mathrm{~cm} / \mathrm{s}$, RI 0.61 . Bilateral prolongation of renal artery acceleration time supports the borderline picture of bilateral RAS. She has undergone PTRA. There was 20-30\% left RAS and $80 \%$ right RAS. A stent was placed on the right renal artery. The patient's condition after PTRA improved, but blood pressure increased at the next follow-up with a mean systolic and diastolic blood pressure of $170 \mathrm{mmHg}$ and $80 \mathrm{mmHg}$, respectively. The patient received four antihypertensive therapies including calcium channel blocker, angiotensin II receptor blocker, and beta-blocker.

\section{Discussion}

In general, RAS is divided into two, namely, ARAS and non-ARAS. Approximately $90 \%$ of RAS is due to atherosclerosis. Atherosclerosis causes stenosis of the $1 / 3$ proximal of the renal arteries, including the perirenal aorta and ostium. Non-ARAS includes fibromuscular dysplasia (FMD), aneurysm, arteriovenous fistula, vasculitis, neurofibromatosis, trauma, embolism, radiation therapy, and vascular dissection. FMD accounts for approximately $10 \%$ of all RAS. Although similar to vasculitis, the exact cause of FMD remains unknown.

The prevalence of RAS in the general population is relatively small. Approximately $16 \%$ of patients with hypertension are thought to have elements of RAS. In the patient population undergoing coronary arteriography, the prevalence increases significantly to $>20 \%[6]$.

ARAS is most common and is especially seen in older patients in the context of systemic atherosclerosis. Many of these plaques are extensions of the aortic plaques into the renal arteries. Therefore, the location of atherosclerotic disease is usually close to the origin of the arteries, although it can be observed anywhere in the renal vessels. Atherosclerosis can affect one or both of the renal arteries. Patients often have other associated risk factors such as diabetes, hypertension, smoking history, peripheral vascular disease, and coronary artery disease (CAD) [7].

The prevalence of RAS is increased in elderly patients, especially in patients with additional comorbid conditions such as diabetes, aortoiliac occlusive disease, CAD, or hypertension.

ARAS of a certain degree is found in $12-45 \%$ of cases in patients with peripheral vascular disease undergoing vascular examination and in $14-40 \%$ of cases in patients undergoing coronary angiography [7].

FMD is a heterogeneous group of nonatherosclerotic and non-inflammatory diseases that affect the intima or fibrous lining of blood vessel walls. Commonly FMD affects the renal and cerebral arteries $(65-70 \%)$ but can affect other sites as well. This can result in arterial stenosis, aneurysm formation with dissection, and/or occlusion of a medium-sized artery. There are two subtypes: Multifocal FMD is the more common form and focal FMD presents as circumferential or tubular stenosis. FMD lesions are usually located far from the origin of the renal artery, often in the center of the vessel or at the first branch of the artery. It occurs mostly in women and is most often diagnosed in middle age [7].

Theoretically, RAS contributes to renovascular hypertension and nephropathy by virtue of its effect on the renin-angiotensin-aldosterone system (RAAS). The RAAS functions to maintain vascular tone, water/salt balance, and heart function through interactions with the sympathetic nervous system and several hormones. It is triggered by hypotension, decreased intravascular volume, hyponatremia, hypokalemia and changes in intravascular volume via $\mathrm{Na}+-\mathrm{K} 2+-\mathrm{Cl}-\mathrm{co}-$ transporters 
at the macula densa in the kidney. After the RAAS is activated, the juxtaglomerular apparatus in the kidney releases renin, which cleaves angiotensinogen to angiotensin I. Angiotensin I is then further cleaved by angiotensin-converting enzyme (ACE) to angiotensin II, which binds to AT1 receptors in the kidney causing efferent arteriolar vasoconstriction, aldosterone stimulation, and increased sodium reabsorption [8].

In RAS, there is decreased perfusion pressure to the kidneys due to RAS, leading to activation of the RAAS. RAAS activation aims to increase renal perfusion by increasing systemic blood pressure. In RAS, vasoconstriction in the efferent glomerular arterioles maintains glomerular filtration in the short term and prevents hypotension and relative hypoxia. While the RAAS provides short-term protection in RAS, in the long term, it causes ischemic nephropathy in the affected kidney, hypertensive nephrosclerosis in the unaffected kidney, and tubular-interstitial glomerulosclerosis and fibrosis in both kidneys. Glomerulosclerosis and tubular interstitial fibrosis are thought to be caused by elevated levels of angiotensin II, which is associated with inflammatory cytokines leading to increased activation of inflammatory and profibrogenic pathways [9].

Additional processes associated with RAS include endothelial dysfunction leading to impaired vascular relaxation, leading to tubular-interstitial fibrosis [10], [11], and increased sympathetic adrenergic activity leading to microvascular damage [10], [11]

\section{Clinical manifestation}

At age $<30$ years, the most likely cause of renovascular hypertension is FMD while at age $>55$ years is ARAS [12]. On laboratory examination, there may be hypokalemia and systolic or diastolic abdominal bruits on physical examination [13].

The most common acute cardiovascular manifestation of renovascular hypertension is flash pulmonary edema, not because of coronary artery or heart valve disease [14]. Other cardiovascular manifestations include hypertension associated acute coronary syndrome, aortic dissection, transient cerebral ischemia, stroke, intracranial hemorrhage, encephalopathy, and papilledema [12]

Renal manifestations associated with RAS include the development of acute renal failure as indicated by an increase in serum creatinine after starting ACE inhibitor or angiotensin II receptor blocker therapy. Acute renal failure often occurs within 10-14 days of starting treatment, but can occur at any time. This finding is considered a classic finding associated with bilateral RAS, but is not sensitive or specific as an indicator of RAS. Other renal manifestations associated with RAS are unexplained chronic renal failure, asymmetric kidney size, and renal atrophy. About $5-15 \%$ of patients who require dialysis annually due to ischemic nephropathy are associated with RAS [15].

\section{Screening/diagnosis}

RAS is often diagnosed incidentally during the evaluation of other arterial diseases, requiring high clinical suspicion to evaluate it. In CKD patients with hypertension, further evaluation is recommended if the onset of the increase in blood pressure occurs before puberty (and precedes the development of CKD), severe or malignant hypertension disproportionate to the degree of CKD and sudden worsening of blood pressure in hypertensive patients who previously had well-controlled hypertension, or the presence of resistant hypertension [5]. Asymmetric kidney size with a difference of $>1.5 \mathrm{~cm}$, an estimated GFR (eGFR) that worsens with ACE inhibitor or angiotensin receptor blockade, the presence of abdominal bruits, or flash pulmonary edema may suggest renovascular hypertension.

The diagnosis of RAS can be made with noninvasive tests including: Captopril-stimulated nuclear renal flow, renal artery duplex ultrasonography, magnetic resonance angiography (MRA), and computerized tomography angiography (CTA) [13]. Each test is an effective diagnostic tool with its own advantages and disadvantages.

Captopril-stimulated nuclear renal flow is used to diagnose unilateral RAS in persons with normal renal function and is considered an effective diagnostic tool. The sensitivity and specificity were $61 \%$ and $86 \%$, respectively [13]. However, in people with bilateral RAS or abnormal kidney function, the accuracy of the test is reduced [13]. Due to its limitations, this test is less preferred as a diagnostic screening tool.

Renal artery duplex ultrasound is considered an effective screening tool and has been validated by many studies. It has a sensitivity between $84-98 \%$, with a specificity between $97-99 \%$ [16], [17]. The diagnosis of RAS is established by measuring the ratio of the PSV in the renal artery compared to the aorta [13]. PSV in the main renal artery is the best parameter to detect significant stenosis. The cutoff PSV value of 180 $200 \mathrm{~cm} / \mathrm{s}$ can detect a reduction in renal artery diameter of $60 \%$. Limitations of ultrasound include body habitus, the pattern of overlying bowel gas that can obscure vision, and the dependence on the role of a sonographer with experience [13]. Renal resistive index $(\mathrm{RI})$ is used in conjunction with renal duplex ultrasonography to evaluate nephropathy. The renal RI is a measurement of the resistance to arterial flow within the renal vessels and is calculated from the arterial Doppler waveform. Renal RI $>0.8$ is considered significant for advanced renal parenchymal disease [18].

Computerized tomography angiography provides higher spatial resolution and is more cost 
effective than MRA, but should be used with caution in patients with impaired renal function due to the use of nephrotoxic iodine contrast. The sensitivity and specificity of MRA were $62 \%$ and $84 \%$, while the sensitivity and specificity of CTA were $64 \%$ and $94 \%$, respectively [19]. Advances in CTA and MRA increased the sensitivity and specificity of CTA to $98 \%$ and $94 \%$ and the sensitivity and specificity of MRA to $100 \%$ and $96 \%$, respectively [20], [21]. The limitations of MRA are its high cost and $10 \%$ of people cannot undergo MRA because of body habitus, metal implants, or claustrophobia. In addition, MRA cannot be used to evaluate the patency of an endoluminal metal stent or the degree of restenosis.

Invasive angiography is the gold standard for the diagnosis of RAS and can be performed by nonselective abdominal aortogram or by selective renal angiography. Reduction of renal artery diameter $>50-70 \%$ leads to reduced blood flow. Unfortunately, reduction in anatomic diameter cannot be used to differentiate which patients would benefit from revascularization [10], [22]. Therefore, the fractional flow reserve (FFR) was measured, which is the ratio of the maximum flow through the lesion compared to the maximum flow without the lesion. To calculate FFR on RAS, the ratio of renal arterial pressure to post-vasodilator aortic pressure was used [21]. FFR $<0.80$ can predict a good blood pressure response to revascularization [12].

\section{Treatment}

Treatment of RAS depends on the type and clinical manifestations. The intervention goals of RAS are to normalize blood pressure, maintain renal function, reduce cardiovascular events, and slow the progression of CKD to kidney transplantation/dialysis. Initial management of RAS is medical/conservative management. Medical therapy is the main pillar of RAS treatment. Its main goals are optimizing glycemic control, lowering blood pressure, lowering cholesterol levels, and modification of risk factors. The recommended antihypertensive drugs for the treatment of RAS are ACE inhibitors, angiotensin receptor blockers, calcium channel blockers, and beta-blockers. ACE inhibitors and angiotensin receptor blockers have been shown to be $86-92 \%$ effective [12].

Indications for conservative therapy: Patients with resistance index values $>80$, pulse pressure $>100$, and advanced nephropathy (protein excretion more than $1 \mathrm{~g} /$ day, creatinine clearance $<40 \mathrm{ml} / \mathrm{min}$, hyperuricemia, pulse pressure $>70 \mathrm{mmHg}$, and no decrease in blood pressure at night) [8] and patients with diameter stenosis $<70 \%$ [23]. Patients with severely reduced renal function who are unlikely to benefit from PTRA include those with CKD Stage 5 and a pole-to-pole kidney size $<7.0 \mathrm{~cm}$ or those receiving hemodialysis therapy for $\geq 3$ months [23].
Interventional therapy is indicated when conservative management fails to control blood pressure, recurrent pulmonary edema occurs, or worsening/chronic azotemia with evidence of significant RAS. In these circumstances, revascularization is considered a treatment option [8]. According to the underlying pathophysiology, it is suspected that revascularization will improve or cure renovascular hypertension and nephropathy, but human studies have not consistently demonstrated these results. The reasons underlying this phenomenon are complex. There are three randomized studies comparing medical therapy with angioplasty with stent placement in patients with ARAS, namely, the Stent Placement in Patients with ARAS and Impaired Renal Function study, Angioplasty and Stenting for Renal Artery Lesions, and Cardiovascular Outcomes with Renal Atherosclerotic Lesions. The results of these studies did not show any difference in mortality, cardiovascular events, or CKD progression [5].

These three studies were criticized for not including a sufficient number of high-risk patients. Whereas most patients not only have a diagnosis of RAS, but also have hypertension and chronic kidney disease. This group of patients is not expected to demonstrate any clinical or measurable benefit from revascularization. It is not surprising, then, that renal revascularization does not produce pathological changes in organs already damaged by chronic hypertension, volume overload, or eGFR-lowering effects. Thus, it may be difficult for studies to demonstrate a major benefit for revascularization, as stents treat only one component of systemic disease [24].

Many experienced clinicians believe that opening the renal arteries in some groups of patients has many advantages over restoring renal blood flow. To date, however, there has been limited evidence to support this perspective. This view is supported by personal clinical experience and retrospective data. ARAS patients who did not undergo revascularization had an adjusted mortality hazard ratio of 1.55 and 2.28 , whereas patients who underwent revascularization had an adjusted mortality ratio of 0.65-0.88 [25].

Several subsets of patients have a clear benefit from revascularization. Revascularization provides benefits in patients with true resistant hypertension based on the results of the ABPM examination (Textor, 2019). Patients who develop acute conditions due to new or occlusive renal artery lesions have the most promise for successful revascularization therapy [24].

Patients who are likely to have improved blood pressure control with revascularization are patients with sudden onset/progression of hypertension, resistant hypertension, and flash pulmonary edema. Several studies have shown a correlation between the presence of resistant hypertension, sudden onset of severe hypertension, and flash pulmonary edema, responding well to revascularization. In addition, 
patients with solitary kidney and RAS or bilateral RAS with worsening renal function may also be considered for revascularization [5].

There has been increasing evidence concerning predictors that may be able to identify RAS patients who will benefit from PTRA. Before conducting PTRA, it is necessary to consider several evaluations. Out-of-office $\mathrm{BP}$ measurement using home BP or ambulatory BP monitoring seems to be crucial. A better BP response to PTRA can be expected in ARAS patients with true resistant hypertension [23]. Assessment of renal function RAS patients showing a rapid renal functional decline is considered suitable candidates for PTRA treatment. Some indicators to predict the clinical course after PTRA are the slope of the reciprocal serum creatinine plot [26], annual rate of eGFR decline [27], and degree of serum creatinine elevation 6 months before PTRA [28], [29]. Renal function should be evaluated based on both eGFR and albuminuria/proteinuria. PTRA has limited benefit for patients with established long-term loss of eGFR, and decreased pre-treatment eGFR and/or increased albuminuria/proteinuria are associated with an increased risk of worse outcomes after PTRA [30]. ARAS patients without pre-treatment albuminuria/proteinuria may be ideal candidates for PTRA treatment [23]. Evaluation of imaging tests including renal ultrasonography and renal RI should be considered before PTRA. Evaluation of kidney morphology should include kidney size and anatomical structure. In cases of atrophic kidney size $(<7 \mathrm{~cm})$ [18], thinning of the renal parenchyma [31], or smaller parenchymal volume to radioisotope-assessed single-kidney GFR [32], it seems that revascularization is not giving the expected improvement. High RI $(>0.8)$ has been suggested to be associated with a worse renal outcome and with poor BP response to revascularization [33].

In general, the first-line revascularization technique in FMD-related RAS is PTRA without stenting, and in ARAS, it is PTRA with stenting. In FMD, revascularization has been shown to be beneficial in reducing hypertension. Research shows that $79 \%$ of patients recover or improve hypertension after revascularization, $65 \%$ of patients maintain this benefit 8 years post-revascularization [34].

In ARAS, revascularization has not been successful in treating renovascular hypertension when compared to FMD. Recovery with normal blood pressure and without medication occurs in $<10 \%$ of patients with ARAS [35] and complete recovery is unlikely. Balloon angioplasty/stenting reduces systolic and diastolic blood pressure when compared with medical therapy alone [36] and decreases the amount of antihypertensive required to control blood pressure [37], [38].

Poor treatment response after revascularization may result from irreversible microvascular disease downstream of the stenotic lesion due to longstanding hypertension. Glomerulosclerosis and nephrosclerosis due to hypertension can increase the blood flow resistance to the affected and unaffected kidneys. Therefore, increased resistance may serve as a marker of renal microvascular structural changes.

Safian and Textor [12] have proposed the following modifications: Patients with no renal parenchymal disease and no hemodynamically significant RAS (renal ischemia) should be medically managed, patients with no renal parenchymal disease but renal ischemia should be considered for revascularization, and patients with renal parenchymal disease are poorer candidates for revascularization and run the risk of having worsening renal function.

Clinicians should recognize that restoration of renal blood flow is effective and important for certain RAS patients. The decision to manage an RAS patient should be highly individualized. However, patient selection is sometimes challenging, due to limited data on the specific phenotypes of patients who would benefit from the procedure [27], [39]. In certain cases, the decision on which therapeutic strategy to pursue remains difficult, but clinicians must evaluate patients carefully individually, to determine whether only optimize medical therapy alone or add PTRA. It is worth considering ARAS as an aspect of atherosclerotic disease, which develops over a longer period of time, and individuals with other comorbidities develop further manifestations of high-grade stenosis and high-risk features.

\section{Conclusion}

RAS is a disease that consists of a broad spectrum of different entities with different pathophysiology that requires varied approaches to diagnose and treat. Current diagnostic tools include MRA, CTA, and renal artery duplex ultrasonography. Patients with renal parenchymal disease are poorer candidates for revascularization. The decision to manage an RAS patient should be highly individualized.

\section{References}

1. Fischer MJ, O'Hare AM. Epidemiology of hypertension in the elderly with chronic kidney disease. Adv Chronic Kidney Dis. 2010;17(4):329-40. https://doi.org/10.1053/j.ackd.2010.05.003 PMid:20610360

2. Riskesdas Board, National Basic Health Research (Riskesdas) Report 2018, Ministry of Health, Indonesia; 2019.

3. IRR. $11^{\text {th }}$ Report of Indonesian Renal Registry 2018; 2018.

4. Sarafidis PA, Persu A, Agarwal R, Burnier M, de Leeuw P, Ferro CJ, et al. Hypertension in dialysis patients: A consensus document by the European renal and cardiovascular medicine (EURECA-m) working group of the European renal 
association-european dialysis and transplant association (ERA-EDTA) and the hypertension and the kidne. Nephrol Dial Transplant. 2017;32(4):620-40. https://doi.org/10.1093/ndt/ gfw433

PMid:28340239

5. Ku E, Lee BJ, Wei J, Weir MR. Hypertension in CKD: Core curriculum 2019. Am J Kidney Dis. 2019;74(1):120-31. https:// doi.org/10.1053/j.ajkd.2018.12.044

PMid:30898362

6. Waafi AK, Samsu N. Renal artery stenosis: Diagnostic and management problems. Clin Res J Intern Med. 2020;1(1):1-13.

7. Herrmann SM, Textor SC. Renovascular hypertension. Physiol Behav. 2019;48(4):765-78. https://doi.org/10.1016/j. ecl.2019.08.007

PMid 31655775

8. Gottam N, Nanjundappa A, Dieter RS. Renal artery stenosis: Pathophysiology and treatment. Expert Rev Cardiovasc Ther. 2009;7(11):1413-20. https://doi.org/10.1586/erc.09.109 PMid:19900024

9. Brewster UC, Setaro JF, Perazella MA. The renin-angiotensinaldosterone system: Cardiorenal effects and implications for renal and cardiovascular disease states. Am JMed Sci. 2003;326(1):1524. https://doi.org/10.1097/00000441-200307000-00003 PMid:12861121

10. Textor SC. Atherosclerotic renal artery stenosis: Overtreated but underrated? J Am Soc Nephrol. 2008;19(4):656-9. https://doi. org/10.1681/ASN.2007111204

PMid:18235081

11. Textor SC. Current approaches to renovascular hypertension. Med Clin North Am. 2009;93(3):717-32. https://doi.org/10.1016/j. mcna.2009.02.012

PMid:19427501

12. Safian RD, Textor SC. Renal-artery stenosis. N Engl J Med. 2001;344(6):431-42. https://doi.org/10.1056/ NEJM200102083440607

PMid:11172181

13. Dieter RS, Schmidt WS, Pacanowski JP, Jaff MR. Renovascular hypertension. Expert Rev Cardiovasc Ther. 2005;3(3):413-22. https://doi.org/10.1586/14779072.3.3.413 PMid:15889969

14. Diamond JR. Flash pulmonary edema and the diagnostic suspicion of occult renal artery stenosis. Am J Kidney Dis. 1993;21(3):328-30. https://doi.org/10.1016/ s0272-6386(12)80756-6

\section{PMid:8447313}

15. Caps MT, Zierler RE, Polissar NL, Bergelin RO, Beach KW, Cantwell-Gab K, et al. Risk of atrophy in kidneys with atherosclerotic renal artery stenosis. J Urol. 1998;53(3):735-42. https://doi.org/10.1046/j.1523-1755.1998.00805.x PMid:9507221

16. Taylor DC, Kettler MD, Moneta GL, Kohler TR, Kazmers A, Beach KW, et al. Duplex ultrasound scanning in the diagnosis of renal artery stenosis: A prospective evaluation. J Vasc Surg. 1988;7(2):363-9.

PMid:3276934

17. Olin JW, Piedmonte MR, Young JR, DeAnna S, Grubb M, Childs MB. The utility of duplex ultrasound scanning of the renal arteries for diagnosing significant renal artery stenosis. Ann Intern Med. 1995;122(11):833-8. https://doi. org/10.7326/0003-4819-122-11-199506010-00004 PMid:7741367

18. Hirsch AT, Haskal ZJ, Hertzer NR, Bakal CW, Creager MA, Halperin JL, et al. ACC/AHA 2005 practice guidelines for the management of patients with peripheral arterial disease (lower extremity, renal, mesenteric, and abdominal aortic): A collaborative report from the american association for vascular surgery/society for vascular surgery, society for cardiovascular angiography and interventions, society for vascular medicine and biology, society of interventional radiology, and the ACC/ AHA task force on practice guidelines (writing committee to develop guidelines for the management of patients with peripheral arterial disease): Endorsed by the american association of cardiovascular and pulmonary rehabilitation; national heart, lung, and blood institute; society for vascular nursing; transatlantic inter-society consensus; and vascular disease foundation. Circulation. 2006;113(11):e463-654. https:// doi.org/10.1161/CIRCULATIONAHA.106.174526 PMid:16549646

19. Vasbinder GB, Nelemans PJ, Kessels AG, Kroon AA, Maki JH, Leiner $\mathrm{T}$, et al. Accuracy of computed tomographic angiography and magnetic resonance angiography for diagnosing renal artery stenosis. Perspect Vasc Surg Endovasc Ther. 2005;17(2):180-2.

20. Postma CT, Joosten FB, Rosenbusch G, Thien T. Magnetic resonance angiography has a artery stenosis high reliability in the detection of renal. In: Haimovici's Vascular Surgery. $6^{\text {th }}$ ed. United States: Wiley; 2012. p. 99-111.

21. Williams GJ, Macaskill P, Chan SF, Karplus TE, Yung W, Hodson EM, et al. Comparative accuracy of renal duplex sonographic parameters in the diagnosis of renal artery stenosis: Paired and unpaired analysis. Am J Roentgenol. 2007;188(3):798-811. https://doi.org/10.2214/AJR.06.0355 PMid:17312071

22. Colyer WR, Cooper CJ, Burket MW, Thomas WJ. Utility of a 0.014 " pressure-sensing guidewire to assess renal artery translesional systolic pressure gradients. Catheter Cardiovasc Interv. 2003;59(3):372-7. https://doi.org/10.1002/ccd.1050 PMid: 12822163

23. Iwashima $Y$, Ishimitsu T. How should we define appropriate patients for percutaneous transluminal renal angioplasty treatment? Hypertens Res. 2020;43(10):1015-27. https://doi. org/10.1038/s41440-020-0496-z

PMid:32572170

24. Colbert GB, Abra G, Lerma EV. Update and review of renal artery stenosis. Dis Month. 2021;67(6):101118. https://doi. org/10.1016/j.disamonth.2020.101118 PMid:33303204

25. Kalra PA, Guo H, Gilbertson DT, Liu J, Chen SC, Ishani A, et al. Atherosclerotic renovascular disease in the United States. Kidney Int. 2010;77(1):37-43. https://doi.org/10.1038/ ki.2009.406 PMid: 19865075

26. Muray S, Martín M, Amoedo ML, García C, Jornet AR, Vera M, et al. Rapid decline in renal function reflects reversibility and predicts the outcome after angioplasty in renal artery stenosis. Am J Kidney Dis. 2002;39(1):60-6. https://doi.org/10.1053/ ajkd.2002.29881

PMid: 11774103

27. Vassallo D, Ritchie J, Green D, Chrysochou C, Kalra PA. The effect of revascularization in patients with anatomically significant atherosclerotic renovascular disease presenting with high-risk clinical features. Nephrol Dial Transplant. 2018;33(3):497-506. https://doi.org/10.1093/ndt/gfx025

PMid:28371854

28. Ritchie J, Green D, Chrysochou C, Chalmers N, Foley RN Kalra PA. High-risk clinical presentations in atherosclerotic renovascular disease: Prognosis and response to renal artery revascularization. Am J Kidney Dis. 2014;63(2):186-97. https:// doi.org/10.1002/ccd.10508

PMid:24074824

29. Kim S, Kim MJ, Jeon J, Jang HR, Park KB, Huh W, et al 
Effects of percutaneous angioplasty on kidney function and blood pressure in patients with atherosclerotic renal artery stenosis. Kidney Res Clin Pract. 2019;38(3):336-46. https://doi. org/10.23876/j.krcp.18.0148

PMid:31234613

30. Kennedy DJ, Colyer WR, Brewster PS, Ankenbrandt M, Burket MW, Nemeth AS, et al. Renal insufficiency as a predictor of adverse events and mortality after renal artery stent placement. Am J Kidney Dis. 2003;42(5):926-35. https://doi. org/10.1016/j.ajkd.2003.06.004

PMid: 14582036

31. Zeller T, Frank U, Müller C, Bürgelin K, Sinn L, Bestehorn HP, et al. predictors of improved renal function after percutaneous stent-supported angioplasty of severe atherosclerotic ostial renal artery stenosis. Circulation. 2003;108(18):2244-9. https:// doi.org/10.1161/01.CIR.0000095786.44712.2A

PMid: 14557357

32. Chrysochou C, Green D, Ritchie J, Buckley DL, Kalra PA. Kidney volume to GFR ratio predicts functional improvement after revascularization in atheromatous renal artery stenosis. PLoS One. 2017;12(6):e0177178. https://doi.org/10.1371/ journal.pone.0177178

PMid:28594847

33. Radermacher J, Chavan A, Bleck J, Vitzthum A, Stoes B. Use of doppler ultrasonography to predict the outcome. N Engl J Med. 2001;344(6):410-7. https://doi.org/10.1056/ NEJM200102083440603

PMid: 11172177

34. Surowiec SM, Sivamurthy N, Rhodes JM, Lee DE, Waldman DL, Green RM, et al. Percutaneous therapy for renal artery fibromuscular dysplasia. Ann Vasc Surg. 2003;17(6):650-5. https://doi.org/10.1007/s10016-003-0070-6

PMid: 14534846

35. Cooper CJ, Murphy TP. Is renal artery stenting the correct treatment of renal artery stenosis? The case for renal artery stenting for treatment of renal artery stenosis. Circulation. 2007;115(2):263-9; discussion 270. https://doi.org/10.1161/ CIRCULATIONAHA.106.619015

PMid:17228012

36. Nordmann AJ, Woo K, Parkes R, Logan AG. Balloon angioplasty or medical therapy for hypertensive patients with atherosclerotic renal artery stenosis? A meta-analysis of randomized controlled trials. Am J Med. 2003;114(1):44-50. https://doi.org/10.1016/ s0002-9343(02)01396-7

PMid: 12557864

37. Plouin PF, Rossignol P, Bobrie G. Atherosclerotic renal artery stenosis: To treat conservatively, to dilate, to stent, or to operate? J Am Soc Nephrol. 2001;12(10):2190-6. https://doi.org/10.1681/ ASN.V12102190

PMid: 11562420

38. van Jaarsveld $B C$, Krijnen $P$, Pieterman $H$, Derkx $F H$, Deinum J, Postma CT, et al. The effect of balloon angioplasty on hypertension in atherosclerotic renal artery stenosis. Curr Hypertens Rep. 2000;2(6):541-2. https://doi.org/10.1056/ NEJM200004063421403

PMid:10749962

39. Karanikola E, Karaolanis G, Galyfos G, Barbaressos E, Palla V, Filis K. Endovascular management of atherosclerotic renal artery stenosis: Post-cardiovascular outcomes in renal atherosclerotic lesions era winner or false alarm? Vasc Spec Int. 2017;33(1):1-15. https://doi.org/10.5758/vsi.2017.33.1.1 PMid:28377906 